\title{
The Evolving Features of the Source Region of a Fast Halo CME with Strong Geo-effects
}

\author{
Zhang Guiqing $\dagger$ \\ National Astronomical Observatories, Chinese Academy of Sciences, Beijing 100012 \\ email: zgq@bao.ac.cn
}

\begin{abstract}
A fast halo CME and correspondingly strong solar burst that occurred in NOAA9684 led to intense geo-effectiveness. The analysis and study in this paper were focused on the evolution of this active region.
\end{abstract}

Keywords. Sun: coronal mass ejections (CMEs), magnetic fields, sunspots

\section{Introduction}

The study of Coronal mass ejections (CMEs) have been one of the most significant topics since they were first observed in the 1970s. The studies of CMEs covered were very extensive. This work tries to analyze and study the evolution of NOAA9684 where a fast halo CME and a strong flare occurred. NOAA9684 was not a high-productive region of remarkable CME and flare. It only produced one remarkable fast Halo CME and an $\mathrm{X} / 3 \mathrm{~b}$ flare during its all-life. The CME that accompanied the X1.0/3B flare erupted on 4 November 2001. This strong eruption led to Sudden Ionospheric Disturbance, proton event with the peak flux of $31700 \mathrm{PFU}(\mathrm{E} \geqslant 10 \mathrm{Mev})$ and a geomagnetic disturbance with a significant $\mathrm{Bz}$ deflection of $-292 \mathrm{nT}$.

\section{Evolving Features of Source Region}

AR9684 appeared on the east limb on 27 October, 2001. It was located at $\mathrm{N} 06^{\circ} \mathrm{L}$ $136^{\circ}$. The leading spot of $\mathrm{N}$ polarity in the active region dominated and its following spot was weak and scattered when it went onto the visible disk (Figure 1(a)).

The Tilt Angle of the Magnetic Axis (TAMA) in a sunspot group has been defined in the studies of Tian (2002). Furthermore, the sign of TAMA and the relation between TAMA and the writhe of a flux tube that formed an active region has been deduced by Fan (1999) and Tian (2002). That is to say, in the northern/southern hemisphere, an Hale active region should rotate clockwise/counter-clockwise and the writhe of magnetic flux tube should be right/left-handed if the rotation appeared in it.

The TAMA of NOAA 9684 was roughly $+2^{\circ}$ on 30 October (Figure $1(\mathrm{a})$ ). The TAMA was roughly $-23^{\circ}$ on 3 November. This case implied that the magnetic flux tube forming the active region rotated $25^{\circ}$ counter-clockwise (left-handedness, (Figure 1(a) and (b)).

We know that the positive/negative values of $\alpha_{\text {best }}$ correspond to the direction of the twist of magnetic lines in right/left-handedness (Tian 2002). We calculated the force-free parameter $\alpha_{\text {best }}$ of NOAA9684 when it was on the heliocentric longitude. It was -0.027 $\pm 0.002 \mathrm{Mm}^{-1}$. This result indicated that the twist of field lines was left-handed in this region.

$\dagger$ The work is supported by NSFC through grants 10073013, 10233050 and 4999-0451. 


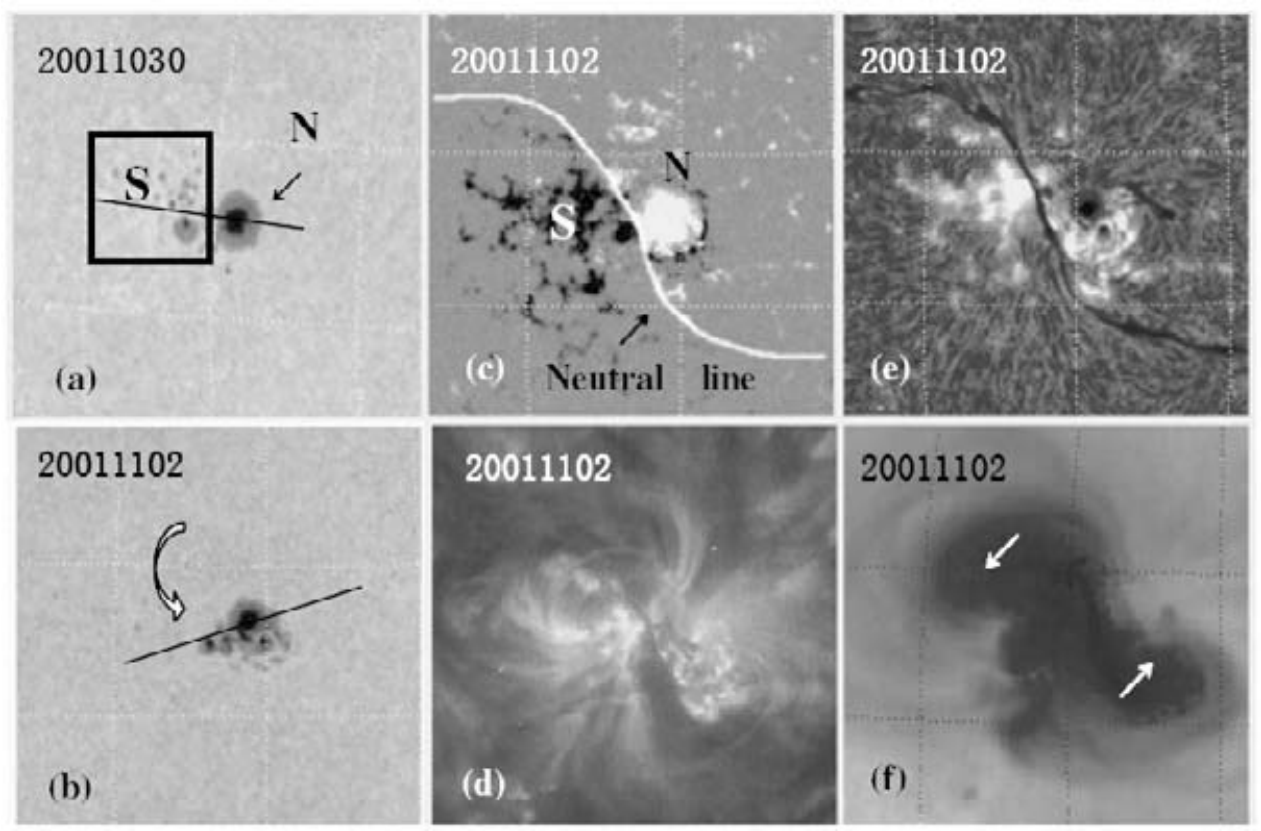

Figure 1. The rotation of sunspot group and "Counter-sigmoid" structure on various layer in NOAA9684. The white and bent arrows indicate the rotating direction of sunspot group and writhing direction of the filament and the loops. The black line indicates the magnetic axis

Figure 1(c) displayed that the magnetic neutral line of NOAA9684 superposed on the neutral line of background magnetic field. Figure 1(d),(e) and (f) are the images of $195 \AA$, $H_{\alpha}$ and soft X-ray. These images showed that the torsion of "Counter-sigmoid" structure of the filament and the magnetic loops are consistent to Counter-clockwise rotation of the active region and the trend of the neutral line of background field.

\section{Discussion and Conclusion}

NOAA9684 was a non-Hale region that disobeyed Joy's law and its maximum area was only $550 \mu \mathrm{h}$. Only one fast Halo CME that accompanied a big flare occurred in it and led to violent geo-effectiveness.

One of the features of the active region was the rotation. The writhe of the magnetic flux tube forming the active region and the twist of the magnetic line were all left-handed, which revealed that the kink instability existed in the active region.

The second feature of NOAA9684 was that its magnetic neutral line superposed on the neutral line of background field, which should indicate that the CME and large flare wonldlead to geo-effectiveness because this location was advantageous to energetic particle flux propagate toward the earth.

\section{References}

Fan, Y., Zweibel, E. G., Linton, M. G. \& Fisher, G. H. 1999, ApJ 521, 460

Tian, L., Liu, Y. \& Wang, J-X. 2002, Solar Phys. 209, 361 